\title{
Ansiedade e Depressão em Vítimas de LCA: Fatores Associados
}

\author{
Anxiety and Depression in ABI Victims: Associated Factors
}

\author{
Ansiedad y Depresión en Víctimas de LCA: \\ Factores Asociados
}

\author{
Célia Marina Mesquita Medeiros ${ }^{1}$, Sandra de Brito Beirão Guerreiro², \\ Maria Vânia Rocha da Silva Nunes ${ }^{3}$
}

\begin{abstract}
1.Mestre em Neuropsicologia, Instituto de Ciências da Saúde da Universidade Católica Portuguesa - ICSUCP. Lisboa, Portugal.

2.Psicóloga no Centro de Reabilitação Profissional de Gaia - CRPG. Vila Nova de Gaia, Portugal. Professora convidada na Universidade Católica Portuguesa, Instituto de Ciências da Saúde (ICS-UCP). Lisboa, Portugal.
\end{abstract}

3.Professora associada da Universidade Católica Portuguesa, Instituto de Ciências da Saúde (ICS-UCP), Centro de Investigação Interdisciplinar em Saúde (CIIS-UCP). Lisboa, Portugal.

\begin{abstract}
Resumo
Introdução. A ansiedade e a depressão constituem os estados emocionais mais frequentemente alterados após Lesão Cerebral Adquirida (LCA). Estas alterações emocionais podem influenciar o progresso de reabilitação e a qualidade de vida destes indivíduos. Objetivo. Identificar fatores sociodemográficos, clínicos e de qualidade de vida associados à sintomatologia ansiosa e depressiva numa população vítima de LCA em contexto de reabilitação neuropsicológica. Método. Trata-se de um estudo correlacional numa amostra de 34 participantes. Foram utilizados um questionário sociodemográfico, o Montreal Cognitive Assessment, o The World Health Organization Quality of Life - Bref, a The Work and Social Adjustment Scale, o Clinical Outcome in Routine Evaluation, e a Hospital Anxiety and Depression Scale. Procedeu-se à análise descritiva dos dados e da relação entre variáveis. Resultados. 55,9\% da amostra apresentou sintomatologia ansiosa, 52,9\% sintomatologia depressiva e $41,2 \%$ comorbidade de sintomas. Os dados sugerem que a probabilidade de indivíduos com LCA desenvolverem sintomas ansiosos aumenta com alterações mais severas da saúde mental global e que a probabilidade de desenvolverem sintomas depressivos é superior nas mulheres cuja qualidade de vida é menor no domínio psicológico. A ocorrência de comorbidade de sintomas é mais provável em mulheres com alterações da saúde mental global e cuja qualidade de vida é menor no domínio psicológico. Conclusão. Reconhecer o papel das dimensões sociodemográficas, clínicas e de qualidade de vida nas alterações emocionais após LCA é fundamental, pois pode auxiliar na identificação de fatores que contribuem para o desenvolvimento de sintomas ansiosos e depressivos.
\end{abstract}

Unitermos. Ansiedade; Depressão; Lesão Cerebral Adquirida; Reabilitação

\footnotetext{
Abstract

Introduction. Anxiety and depression are the most frequently altered emotional states after Acquired Brain Injury (ABI). These emotional changes can influence the progress of rehabilitation and the quality of life of these individuals. Objective. Identify sociodemographic, clinical, and quality of life factors associated with anxious and depressive symptoms in an ABI victim population in the context of neuropsychological rehabilitation. Method. This is a correlational study in a sample of 34 participants. A sociodemographic questionnaire, the Montreal Cognitive Assessment, The World Health Organization Quality of Life - Bref, The Work and Social Adjustment Scale, the Clinical Outcome in Routine Evaluation, and the Hospital Anxiety and Depression Scale were used. A descriptive analysis of the data and the relationship between variables was carried out. Results. $55.9 \%$ of the sample had anxious symptoms, $52.9 \%$ depressed symptoms and $41.2 \%$ symptom comorbidity. The data suggest that the probability of individuals with $A B I$ to develop anxiety symptoms increases with more severe
} 
changes in global mental health and that the probability of developing depressive symptoms is higher in women whose quality of life is lower in the psychological domain. The occurrence of symptom comorbidity is more likely in women with changes in global mental health and whose quality of life is lower in the psychological domain. Conclusion. Recognizing the sociodemographic, clinical, and quality of life dimensions in detecting emotional changes after ABI is essential, as it can assist in the identification of factors that contribute to the development of anxious and depressive symptoms.

Keywords. Anxiety; Depression; Acquired Brain Injury; Rehabilitation

\section{Resumen}

Introducción. La ansiedad y la depresión son los estados emocionales que se alteran con mayor frecuencia después de una Lesión Cerebral Adquirida (LCA). Estos cambios emocionales pueden ser determinantes en el avance de la rehabilitación y en la calidad de vida de estos individuos. Objetivo. Identificar factores sociodemográficos, clínicos y de calidad de vida asociados con síntomas ansiosos y depresivos en una población víctima de LCA en el contexto de rehabilitación neuropsicológica. Método. Este es un estudio correlacional en una muestra de 34 participantes. Se utilizó un cuestionario sociodemográfico, el Montreal Cognitive Assessment, el The World Health Organization Quality of Life - Bref, la The Work and Social Adjustment Scale, el Clinical Outcome in Routine Evaluation y la Hospital Anxiety and Depression Scale. Se realizó un análisis descriptivo de los datos y la relación entre variables. Resultados. $55.9 \%$ de la muestra presentó síntomas ansiosos, 52.9\% síntomas depresivos y $41.2 \%$ comorbilidad sintomática. Los datos sugieren que la probabilidad de que los individuos con LCA desarrollen síntomas de ansiedad aumenta con cambios más severos en la salud mental global y que la probabilidad de desarrollar síntomas depresivos es mayor en las mujeres cuya calidad de vida es menor en el dominio psicológico. La aparición de comorbilidad de síntomas es más probable en mujeres con cambios en la salud mental global y cuya calidad de vida es menor en el dominio psicológico. Conclusión. Reconocer las dimensiones sociodemográficas, clínicas y de calidad de vida en la detección de cambios emocionales después del LCA es fundamental, ya que puede ayudar en la identificación de factores que contribuyen al desarrollo de síntomas ansiosos y depresivos.

Palabras clave. Ansiedad; Depresión; Lesión Cerebral Adquirida; Rehabilitación

Trabalho realizado no Instituto de Ciências da Saúde da Universidade Católica Portuguesa, Lisboa, Portugal.

Endereço para correspondência: Maria Vânia Rocha da Silva Nunes. Universidade Católica Portuguesa. Palma de Cima, 1649-023. Lisboa, Portugal. E-mail: mnunes@ucp.pt

\section{INTRODUÇÃO}

Os estudos da área da neuropsicologia das emoções tiveram um crescimento exponencial nos últimos 30 anos, apesar disso este conhecimento ainda não está totalmente integrado na prática da neuropsicologia clínica, observandose uma imprecisão nos protocolos de avaliação da sintomatologia ansiosa e depressiva na área da reabilitação neuropsicológica ${ }^{1}$. 
De acordo com a literatura, após uma Lesão Cerebral Adquirida (LCA) é frequente ocorrerem alterações emocionais $^{2}$, sendo a ansiedade e a depressão os estados emocionais mais frequentemente alterados ${ }^{2}$. Sabe-se que estas alterações emocionais tendem a influenciar a qualidade de vida de forma negativa e a piorar os resultados de reabilitação ${ }^{3,4}$. A prevenção destas alterações poderá surgir de um diagnóstico mais preciso, identificando os fatores que podem estar associados a sintomas ansiosos, depressivos e comorbidade entre estes sintomas.

As LCA constituem um grupo específico das doenças neurológicas, abarcando todas as lesões que representam qualquer dano nas estruturas cerebrais e que ocorrem em algum momento após o nascimento. Em Portugal, o Acidente Vascular Cerebral (AVC) e o Traumatismo Cranioencefálico (TCE) constituem as causas mais comuns de LCA ${ }^{5,6}$.

Na sequência de uma LCA é comum ocorrerem danos no funcionamento físico, cognitivo, comportamental e/ou emocional, que resultam em limitações em determinadas atividades. Os indivíduos com LCA tendem a apresentar alterações emocionais, em parte, devido a mudanças anatômicas cerebrais específicas ${ }^{2}$. No entanto, estas alterações emocionais também podem surgir como uma reação às mudanças nas circunstâncias ${ }^{2,7}$. Neste sentido, é frequente pessoas com LCA desenvolverem sentimentos negativos, pois há um descontentamento com a situação em que se encontram. Entre as consequências diretas mais 
comuns da LCA nas alterações emocionais estão a ansiedade aumentada e humor deprimido².

A ansiedade consiste na antecipação de uma ameaça futura, caracterizada como um estado emocional de medo ou receio constante e preocupação excessiva desproporcionados à situação real ${ }^{8}$. Já a depressão pode ser considerada como um estado emocional persistente de baixa autoestima, tristeza e desesperança, no que diz respeito ao mundo, ao futuro e à própria pessoa ${ }^{9}$.

De acordo com um estudo neerlandês ${ }^{10}$ a incidência de sintomatologia ansiosa e de sintomatologia depressiva em doentes com LCA foi de 30,8\% e 36,7\%, respectivamente. Para avaliar estas sintomatologias, os autores recorreram à Hospital Anxiety and Depression Scale (HADS), utilizando o ponto de corte 8. A amostra final foi constituída por 122 participantes (76 do gênero masculino), com média $54,4 \pm 12,2$ anos de idade. A maioria das pessoas da amostra tinha sido vítima de eventos vasculares $(n=76)$, sendo 0 tempo médio da lesão cerebral de $14,1 \pm 8,6$ semanas. Neste estudo foram excluídos os participantes que apresentassem qualquer doença cerebral progressiva pré-mórbida, domínio insuficiente da língua neerlandesa, e incapacidade para o preenchimento de questionários com base no julgamento clínico (afasia e comprometimento cognitivo grave).

Apesar de existirem alguns estudos na área das alterações emocionais em vítimas de LCA, estes não apresentam padrões consistentes no que diz respeito à associação de fatores às sintomatologias ansiosa e 
depressiva, sendo mais evidente a controvérsia de resultados no que diz respeito à sintomatologia ansiosa.

Tendo em consideração a literatura que aborda a associação da sintomatologia ansiosa a fatores sociodemográficos: gênero e idade, é possível perceber pelos resultados destes estudos que nenhum destes fatores apresentou uma associação significante com a sintomatologia ansiosa ${ }^{11,12}$. No entanto, segundo a literatura $^{12-15}$ que aborda a associação da sintomatologia depressiva a fatores sociodemográficos: gênero ${ }^{14,15}$, idade $^{12,14,15}$, estado civil ${ }^{15}$, escolaridade ${ }^{14}$ e situação face ao emprego ${ }^{13}$, é possível perceber pelos resultados que há uma controvérsia relativamente aos fatores gênero ${ }^{14,15} \mathrm{e}$ idade $12,14,15$, havendo autores que confirmam uma associação e outros que não. Relativamente ao estado civil ${ }^{15}$, os resultados da literatura de referência não apresentaram uma associação à sintomatologia depressiva. Apenas os fatores escolaridade ${ }^{14}$ e situação face ao emprego ${ }^{13}$ apresentaram uma associação significante com a sintomatologia depressiva na literatura de referência. Estes estudos verificaram que a incidência de sintomatologia depressiva foi superior nos indivíduos com escolaridade inferior a 10 anos e, também, naqueles com desemprego prévio à lesão.

De acordo com a literatura ${ }^{3,11,16-19}$ que aborda a associação da sintomatologia ansiosa a fatores clínicos: localização da lesão ${ }^{11,16-19}$, funções cognitivas ${ }^{16,20}$, capacidades funcionais ${ }^{3,21}$, autonomia nas Atividades de Vida 
Diária (AVD) ${ }^{11}$ e qualidade de vida $3,19,20$, é possível verificar pelos resultados destes estudos que há uma controvérsia no que diz respeito aos fatores localização da lesão ${ }^{11,16-19} \mathrm{e}$ funções cognitivas ${ }^{16,20}$, havendo autores que confirmam uma associação e outros que não. Apenas os fatores capacidades funcionais ${ }^{3,21}$ e qualidade de vida ${ }^{3,19,20}$ apresentaram uma associação significante com a sintomatologia ansiosa na literatura de referência. Estes estudos verificaram que a incidência de sintomatologia ansiosa foi superior nos indivíduos com capacidades funcionais mais reduzidas e, ainda, naqueles com uma qualidade de vida mais baixa. No que diz respeito à autonomia nas $\mathrm{AVD}^{11}$ os resultados da literatura de referência não apresentaram uma associação à sintomatologia ansiosa.

Ainda no que diz respeito aos fatores clínicos, de acordo com a literatura ${ }^{3,13-16,18,20-23}$ que aborda a associação destes fatores à sintomatologia depressiva, é possível verificar pelos resultados destes estudos que há uma controvérsia no que diz respeito aos fatores localização da lesão ${ }^{14,16,18,22}$ e funções cognitivas ${ }^{14,15}$, havendo autores que confirmam uma associação e outros que não. Apenas os fatores: capacidades funcionais ${ }^{3,13-15,21}$, autonomia nas $\operatorname{AVD}^{3,13,15}$ e qualidade de vida $^{3,14,20,23}$ apresentaram uma associação significante com a sintomatologia depressiva na literatura de referência. Estes estudos verificaram que a incidência de sintomatologia depressiva foi superior nos indivíduos com capacidades funcionais mais reduzidas, naqueles com menor autonomia 
nas AVD, e, ainda, naqueles com uma qualidade de vida mais baixa.

De acordo com o que foi referido anteriormente na revisão da literatura, é possível concluir que há uma escassa realização de estudos, tanto nacionais como estrangeiros, sobre a relação entre alterações emocionais após LCA e fatores associados, e por isto torna-se essencial a realização de novos estudos de investigação acerca desta temática.

Conforme supramencionado, os resultados de reabilitação tendem a ser influenciados negativamente pelas alterações emocionais em indivíduos com LCA ${ }^{3,4}$. Neste sentido, as alterações emocionais e de humor, habitualmente, constituem o obstáculo mais duradouro e problemático à reinserção do indivíduo com LCA na comunidade ${ }^{7}$. Os centros de reabilitação que recebem e respondem às necessidades de indivíduos com LCA disponibilizam programas de reabilitação neuropsicológica capazes de articular terapias individuais, de grupo e baseadas na comunidade, abordando todas as problemáticas do indivíduo, e possibilitando alcançar uma recuperação completa ou o expoente máximo possível das suas capacidades físicas, mentais e sociais, reduzindo o impacto dos seus problemas na vida cotidiana e promovendo a sua reintegração nos contextos em que estão incluídos ${ }^{24}$. Mesmo tratando-se de uma área de atuação desafiadora, a reabilitação da componente emocional é reconhecida como uma das principais áreas de desenvolvimento nos serviços neurológicos ${ }^{9}$. 
Posto isto, o presente estudo teve como principal foco a saúde mental de indivíduos com LCA. Pretendeu-se, nomeadamente, analisar a incidência de sintomatologia ansiosa e de sintomatologia depressiva nesta população específica e que se encontra em contexto de reabilitação neuropsicológica, visando a identificação de fatores sociodemográficos ( gênero, idade, estado civil, escolaridade e situação face a emprego), clínicos ( tempo pós-lesão, tipo de lesão, gravidade da lesão, localização da lesão, tipo e gravidade de alterações nas funções, e autonomia na realização de atividades de vida diária) e de qualidade de vida associados.

\section{MÉTODO}

Trata-se de um estudo correlacional, aprovado pela Comissão de Ética para a Saúde de uma universidade portuguesa em 22 de outubro de 2020, com o parecer do projeto número 81 .

\section{Amostra}

O processo de amostragem do presente estudo foi por conveniência, sendo esta amostra constituída por indivíduos que frequentam um Centro de Reabilitação, incluídos ou a incluir num Programa Holístico de Reabilitação Neuropsicológica (PHRN). Foram incluídas no estudo todas as pessoas com idade igual ou superior a 18 anos e previamente diagnosticadas com LCA resultante de TCE, 
AVC, tumores ou outros. Visto que a capacidade de compreender e responder aos questionários é determinante para possibilitar uma análise fidedigna dos dados, estiveram excluídas do estudo as pessoas que apresentassem: 1) perturbações mentais severas e/ou 2) afasia severa.

A amostra final foi composta por 34 participantes (22 do gênero masculino). A idade média é de $44,1 \pm 12,6$ anos e

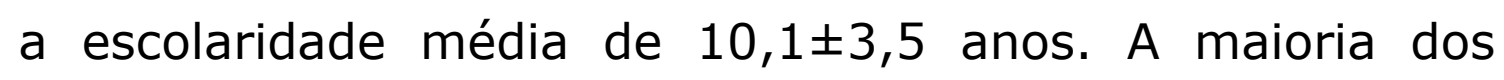
participantes foi diagnosticado quando da lesão com gravidade ligeira a moderada $(n=31)$, sendo o tempo médio decorrido após a lesão de 2,2 $\pm 3,0$ anos, o mecanismo da lesão majoritariamente composto por eventos vasculares $(n=20)$, e a localização da lesão, em metade dos participantes, localizada no hemisfério cerebral direito $(n=17)$. Para além disto, a maioria dos participantes referiu ser totalmente autônomo nas AVD $(N=24)$, como é apresentado na Tabela 1.

\section{Procedimento}

O protocolo de recolhimento de dados foi administrado individualmente, num único momento e com uma sequência pré-definida, com uma duração aproximada de 30 a 45 minutos para cada participante.

O estudo foi apresentado a cada um dos participantes, de forma a expor os principais objetivos, esclarecer a participação voluntária e a garantir a confidencialidade das informações recolhidas ao longo do estudo. 
Tabela 1. Características sociodemográficas e clínicas da amostra $(N=34)$.

\begin{tabular}{lcc}
\hline Variáveis & Média $\pm \boldsymbol{D P}$ & $\boldsymbol{n}(\mathbf{\%})$ \\
\hline Gênero & $22(64,7)$ \\
Masculino & $12(35,3)$ \\
Feminino & $44,1 \pm 12,6$ & \\
\hline Idade & $10,1 \pm 3,5$ & \\
\hline Escolaridade & $14(41,2)$ \\
\hline Estado civil & $16(47,1)$ \\
Solteiro/a & $4(11,8)$ \\
Casado/a & \\
Divorciado/a & $8(23,5)$ \\
\hline Situação face ao emprego & $12(35,3)$ \\
Sem ocupação laboral prévia & $14(41,2)$ \\
Sem ocupação laboral posterior & \\
Com ocupação laboral & $2,2 \pm 3,0$ \\
\hline Tempo pós-lesão (anos) & $20(58,8)$ \\
\hline Mecanismo da lesão & $4(11,8)$ \\
Eventos vasculares & $10(29,4)$ \\
Eventos traumáticos & \\
Outras causas & $31(91,2)$ \\
\hline Gravidade da lesão & $3(8,8)$ \\
Ligeira a moderada & $7(20,6)$ \\
Severa & $17(50,0)$ \\
\hline Localização da lesão & $5(14,7)$ \\
Hemisfério cerebral esquerdo & \\
Hemisfério cerebral direito & \\
Difusa & \\
\hline Autonomia nas AVD & \\
Totalmente autônomo & \\
Independência modificada & \\
Necessidade de apoio 3a pessoa & \\
\hline
\end{tabular}

Neste seguimento, foi aplicado o Termo de Consentimento Livre e Esclarecido. Posteriormente, foi utilizado o Questionário Sociodemográfico para recolher os dados relativos à caracterização da amostra. Em seguida, 
foram utilizados os instrumentos adequados para a avaliação neuropsicológica.

O Montreal Cognitive Assessment (MoCA) ${ }^{25}$, com excelente consistência interna (alfa de Cronbach de 0,92) ${ }^{25}$, foi utilizado para aferir a presença de déficit cognitivo e respectiva severidade. No total, esta medida de avaliação, é constituída por 15 tarefas com uma cotação máxima de 30 pontos ${ }^{25}$. Para aferir a presença de déficit cognitivo, recorreuse aos valores normativos das pontuações do MoCA de acordo com a idade e a escolaridade de cada participante, considerando as pontuações de $1 D P$ como déficit cognitivo ligeiro, $1,5 D P$ como déficit moderado e $2 D P$ ou mais como déficit cognitivo grave.

O World Health Organization Quality of Life - Bref (WHOQOL-BREF, versão breve) ${ }^{26}$, com boa consistência interna (alfa de Cronbach a variar entre 0,64 e 0,87$)^{26}$, foi utilizado para avaliar a qualidade de vida. Trata-se de um questionário de autopreenchimento constituído por 26 itens e com pontuações dimensionadas para variar de 0 a 100 para cada domínio (físico, psicológico, social e ambiente) ${ }^{26}$. Quanto maior for a pontuação do domínio, maior será a qualidade de vida e a saúde naquele domínio ${ }^{26}$.

A Work and Social Adjustment Scale (WSAS) ${ }^{27}$, com boa consistência interna (alfa de Cronbach a variar entre 0,70 e $0,94)^{27}$, foi utilizada para avaliar o comprometimento funcional atribuível a um problema identificado. Trata-se de uma escala de autopreenchimento constituída por 5 itens e com uma pontuação máxima de 40 pontos, sendo que 
pontuações mais elevadas correspondem a piores resultados ${ }^{27}$.

O Clinical Outcome in Routine Evaluation (CORE-10, versão de 10 itens) ${ }^{28}$, com excelente consistência interna (alfa de Cronbach superior a 0,80$)^{28}$, foi utilizado como medida breve de triagem do bem-estar psicológico em adultos. Trata-se de um instrumento de autopreenchimento, constituído por 10 itens e com uma pontuação máxima de 40 pontos, sendo que pontuações mais elevadas correspondem a maior sentimento de angústia ${ }^{28}$.

A Hospital Anxiety and Depression Scale (HADS) ${ }^{29}$, com boa consistência interna (alfa de Cronbach de 0,76 para a subescala de ansiedade e de 0,81 para a subescala de depressão) $)^{29}$, foi utilizada como medida de triagem de ansiedade e depressão clinicamente significativas. Trata-se de uma escala de autopreenchimento constituída por 14 itens e com uma pontuação máxima de 21 pontos para cada subescala (ansiedade e depressão) ${ }^{29}$. De um modo geral, pontuações iguais ou superiores a 8 podem ser consideradas como implicando a presença de perturbação do humor ${ }^{29}$.

\section{Análise Estatística}

A análise estatística dos dados foi realizada através da versão 25.0 do software IBM Statistical Package for the Social Sciences (SPSS), recorrendo à análise descritiva, à análise de associação, através do teste de Qui-quadrado e do teste exato de Fisher, e à análise de regressão logística. 
Considerou-se, por defeito, um nível de significância de $p<, 05$ para resultados significantes.

De forma a facilitar a análise dos resultados, as variáveis escalares foram reorganizadas em classes: idade, segundo a análise de frequências; escolaridade, segundo o sistema educativo português; e tempo pós-lesão, tendo em consideração a média.

\section{RESULTADOS}

\section{Análise da incidência de sintomatologia}

Os resultados relativos à avaliação da sintomatologia ansiosa e depressiva são apresentados na Tabela 2.

Tabela 2. Distribuição da incidência de sintomatologia ansiosa, sintomatologia depressiva e comorbidade entre sintomatologias em pessoas com LCA em contexto de reabilitação.

\begin{tabular}{lcc}
\hline & $\boldsymbol{M} \pm \boldsymbol{D P}$ & $\boldsymbol{n}(\mathbf{\%})$ \\
\hline Sintomatologia ansiosa (HADS) & $8,1 \pm 5,3$ & $19(55,9)$ \\
Sintomatologia depressiva (HADS) & $7,0 \pm 4,7$ & $18(52,9)$ \\
Sintomatologia ansiosa e depressiva (HADS) & & $14(41,2)$ \\
\hline
\end{tabular}

De acordo com os resultados, a incidência de sintomas ansiosos foi de $55,9 \%(n=19)$, de sintomas depressivos foi de $52,9 \% \quad(n=18)$, e de comorbidade entre sintomas ansiosos e depressivos foi de $41,2 \%(n=14)$. 


\section{Análise da avaliação neuropsicológica}

Os resultados da avaliação neuropsicológica são apresentados na Tabela 3.

Tabela 3. Resultados da avaliação neuropsicológica $(N=34)$.

\begin{tabular}{lcc}
\hline & $\boldsymbol{M} \pm \boldsymbol{D P}$ & $\boldsymbol{n}(\mathbf{\%})$ \\
\hline Funcionamento cognitivo global (MoCA) & $21,1 \pm 6,0$ & \\
Capacidades funcionais (WSAS) & $20,1 \pm 9,0$ & \\
Saúde mental global (CORE-10) & $16,5 \pm 72$ & \\
Qualidade de vida global (WHOQOL-BREF) & & $2(5,9)$ \\
$\quad$ Má & & $14(41,2)$ \\
$\quad$ Nem boa nem má & & $15(44,1)$ \\
Boa & & $3(8,8)$ \\
Muito boa & $60,9 \pm 16,8$ & \\
Qualidade de vida DF (WHOQOL-BREF) & $60,3 \pm 22,7$ & \\
Qualidade de vida DP (WHOQOL-BREF) & $59,6 \pm 231$ & \\
Qualidade de vida DS (WHOQOL-BREF) & $63,2 \pm 15,9$ \\
Qualidade de vida DA (WHOQOL-BREF) & & \\
\hline
\end{tabular}

DF = Domínio Físico; DP = Domínio Psicológico; DS = Domínio Social; DA = Domínio Ambiente

A avaliação do funcionamento cognitivo global (MoCA) teve uma média de $21,1 \pm 6,0$ pontos, sendo que $41,2 \%$ $(n=14)$ dos participantes apresentou alterações cognitivas severas. No que diz respeito à avaliação das capacidades

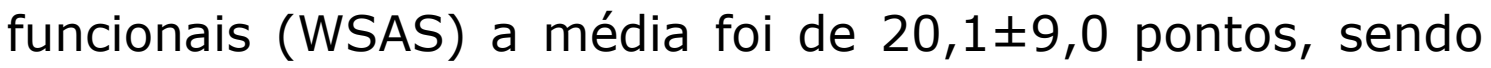
que 55,9\% $\quad(n=19)$ evidenciou alterações severas. A avaliação da saúde mental global (CORE-10) teve uma média de $16,5 \pm 7,2$ pontos, sendo que 29,4\% $(n=10)$ apresentou um sentimento de angústia ligeiro. A avaliação da qualidade de vida (WHOQOL-BREF) revelou que 44,1\% $(n=15)$ dos participantes considerou a sua qualidade de vida como boa. 
Relativamente aos domínios específicos da qualidade de vida, em média, os participantes revelaram uma percepção positiva da qualidade de vida: DF com $60,9 \pm 16,8$ pontos, DP com 60,3 $\pm 22,7$ pontos, DS com 59,6 $\pm 23,1$ pontos e DA com $63,2 \pm 15,9$ pontos.

\section{Análise da associação entre variáveis}

A comparação entre pessoas vítimas de LCA com e sem sintomatologia ansiosa, sintomatologia depressiva, e ambas as sintomatologias foi realizada tendo em consideração as características sociodemográficas, clínicas e de qualidade de vida desses indivíduos.

No que diz respeito às características sociodemográficas a sintomatologia ansiosa teve maior incidência nos participantes do gênero masculino $(52,6 \%)$, nos participantes com 35 a 47 anos $(31,6 \%)$ e 48 a 54 anos $(31,6 \%)$ de idade, nos participantes com 10 a 12 anos $(47,4 \%)$ de escolaridade, nos participantes solteiros $(47,4 \%)$, e nos participantes com ocupação laboral $(50,0 \%)$. Contudo, as variáveis: gênero $(p=0,097)$, idade $(p=0,569)$, escolaridade $(p=0,152)$ e situação face ao emprego $(p=1,000)$ não apresentaram uma associação significante com os sintomas ansiosos.

Relativamente às características clínicas, a incidência de sintomatologia ansiosa foi superior nos participantes com lesão cerebral de 1 a 3 anos (47,4\%), nos participantes cuja lesão cerebral foi desencadeada por eventos vasculares $(57,9 \%)$, nos participantes com lesão cerebral de gravidade 
ligeira a moderada $(89,5 \%)$ e nos participantes com lesão cerebral no hemisfério cerebral direito $(47,4 \%)$. No entanto, nenhuma destas variáveis: tempo pós-lesão $(p=1,000)$, mecanismo da lesão $(p=1,000)$, gravidade da lesão $(p=1,000)$ e localização da lesão $(p=1,000)$ apresentou uma associação significante com os sintomas ansiosos.

A sintomatologia ansiosa apresentou, ainda, maior prevalência nos participantes com alterações cognitivas severas $(52,6 \%)$, nos participantes com alterações severas das capacidades funcionais $(73,7 \%)$, nos participantes com alterações moderadas $(31,6 \%)$ e severas $(31,6 \%)$ da saúde mental global, e nos participantes totalmente autônomos nas AVD $(63,2 \%)$. No entanto, as variáveis: funcionamento cognitivo global $(p=0,450)$ e autonomia nas AVD $(p=0,654)$ não apresentaram associação significante com os sintomas ansiosos.

No que diz respeito à qualidade de vida a sintomatologia ansiosa teve maior incidência nos participantes com uma qualidade de vida global intermédia $(47,4 \%)$, nos participantes com uma qualidade de vida intermédia no DF $(36,8 \%)$, nos participantes com uma qualidade de vida boa no DP $(36,8 \%)$, nos participantes com uma qualidade de vida boa no DS $(57,9 \%)$, e nos participantes com uma qualidade de vida intermédia $(36,8 \%)$ e boa $(36,8 \%)$ no DA. No entanto, a qualidade de vida global $(p=0,138)$, o DF $(p=0,084)$ e o DA $(p=0,488)$ não apresentaram associação significante com os sintomas ansiosos. 
Assim, verifica-se que apenas as capacidades funcionais $(p=0,017)$, a saúde mental global $(p=0,002)$, o DP $(p=0,032)$ e DS $(p=0,010)$ da qualidade de vida, apresentaram associação significante com os sintomas ansiosos.

No que diz respeito às características sociodemográficas, a sintomatologia depressiva teve maior incidência nos participantes do gênero feminino $(55,6 \%)$, nos participantes de 18 a 34 anos $(38,9 \%)$ de idade, nos participantes com 10 a 12 anos $(50,0 \%)$ de escolaridade, nos participantes solteiros $(50,0 \%)$, e nos participantes sem ocupação laboral posterior à lesão (38.9\%). No entanto, as variáveis: idade $(p=0,226)$, escolaridade $(p=0,478)$, estado civil $(p=0,343)$, e situação face ao emprego $(p=0,745)$ não apresentaram associação significante com os sintomas depressivos.

Relativamente às características clínicas, a sintomatologia depressiva teve maior incidência nos participantes com lesão cerebral de 1 a 3 anos $(61,1 \%)$, nos participantes cuja lesão cerebral foi desencadeada por eventos vasculares $(50,0 \%)$, nos participantes com lesão cerebral de gravidade ligeira a moderada $(83,3 \%)$, e nos participantes com lesão cerebral no hemisfério cerebral direito $(44,4 \%)$. Contudo, nenhuma destas variáveis: tempo pós-lesão $(p=0,265)$, mecanismo da lesão $(p=0,168)$, gravidade da lesão $(p=0,230)$, e localização da lesão $(p=0,903)$ apresentou associação significante com os sintomas depressivos. 
A sintomatologia depressiva apresentou, ainda, maior incidência nos participantes com alterações cognitivas severas $(50,0 \%)$, nos participantes com alterações severas das capacidades funcionais $(72,2 \%)$, nos participantes com alterações severas da saúde mental global (33,3\%), e nos participantes totalmente autônomos nas AVD (66,7\%). No entanto, nenhuma destas variáveis: funcionamento cognitivo global $(p=0,456)$, capacidades funcionais $(p=0,173)$, saúde mental global $(p=0,090)$, e autonomia nas AVD $(p=0,562)$ apresentou associação significante com os sintomas depressivos.

No que diz respeito à qualidade de vida, a sintomatologia depressiva teve maior incidência nos participantes com uma qualidade de vida global intermédia $(44,4 \%)$ e boa $(44,4 \%)$, nos participantes com uma qualidade de vida boa no DF $(38,9 \%)$, nos participantes com uma qualidade de vida boa no DP $(33,3 \%)$, nos participantes com uma qualidade de vida intermédia no DS $(55,6 \%)$, e nos participantes com uma qualidade de vida intermédia $(33,3 \%)$ e boa $(33,3 \%)$ no DA. Contudo, a qualidade de vida global $(p=0,213)$, o $\operatorname{DF}(p=0,086)$ e o $\mathrm{DA}(p=0,681)$ não apresentaram associação significante com os sintomas depressivos.

Assim, verifica-se que apenas o gênero $(p=0,009)$, 0 DP $(p=0,029)$ e DS $(p=0,032)$ da qualidade de vida, apresentaram associação significante com os sintomas depressivos. 
No que diz respeito às características sociodemográficas, a comorbidade de sintomatologias teve maior incidência nos participantes do gênero feminino $(64,3 \%)$, nos participantes com 18 a 34 anos $(35,7 \%)$ e 35 a 47 anos $(35,7 \%)$ de idade, nos participantes com 10 a 12 anos $(57,1 \%)$ de escolaridade, nos participantes solteiros $(50,0 \%)$, e nos participantes tanto sem ocupação laboral posterior à lesão cerebral $(35,7 \%)$ como com ocupação laboral $(35,7 \%)$. No entanto, as variáveis: idade $(p=0,248)$, escolaridade $(p=0,227)$, estado civil $(p=0,699)$, e situação face ao emprego $(p=0,904)$ não apresentaram associação significante com a comorbidade de sintomas.

Relativamente às características clínicas, a comorbidade de sintomatologias teve maior incidência nos participantes com lesão cerebral de 1 a 3 anos $(57,1 \%)$, nos participantes cuja lesão cerebral foi desencadeada por eventos vasculares $(50,0 \%)$, nos participantes com lesão cerebral de gravidade ligeira a moderada $(85,7 \%)$, e nos participantes com lesão cerebral no hemisfério cerebral direito (42,9\%). Contudo, nenhuma destas variáveis: tempo pós-lesão $(p=0,889)$, mecanismo da lesão $(p=0,607)$, gravidade da lesão $(p=0,555)$, e localização da lesão $(p=0,897)$ apresentou associação significante com a comorbidade de sintomas.

A comorbidade de sintomatologias apresentou, ainda, maior incidência nos participantes com alterações cognitivas severas $(57,1 \%)$, nos participantes com alterações severas das capacidades funcionais $(78,6 \%)$, nos participantes com 
alterações severas da saúde mental global (42,9\%), e nos participantes totalmente autônomos nas AVD (64,3\%). No entanto, as variáveis: funcionamento cognitivo global $(p=0,260)$, capacidades funcionais $(p=0,058)$, e autonomia nas AVD $(p=0,859)$ não apresentaram associação significante com a comorbidade de sintomas.

No que diz respeito à qualidade de vida, a comorbidade de sintomatologias teve maior incidência nos participantes com uma qualidade de vida global intermédia (42,9\%) e boa $(42,9 \%)$, nos participantes com uma qualidade de vida boa no DF $(35,7 \%)$, nos participantes com uma qualidade de vida má $(28,6 \%)$ e boa $(28,6 \%)$ no DP, nos participantes com uma qualidade de vida intermédia no DS (64,3\%), e nos participantes com uma qualidade de vida intermédia no DA $(35,7 \%)$. Contudo, a qualidade de vida global $(p=0,256)$ e 0 DA $(p=0,265)$ não apresentaram associação significante com a comorbidade de sintomas.

Assim, verifica-se que apenas o gênero $(p=0,005)$, a saúde mental global $(p=0,007)$, o DF $(p=0,013)$, DP $(p=0,008)$ e DS $(p=0,024)$ da qualidade de vida, apresentaram associação significante com a comorbidade de sintomas ansiosos e depressivos.

\section{Análise da regressão logística}

A regressão logística foi realizada para cada uma das sintomatologias tendo como variáveis independentes aquelas que apresentaram associação significante $(p<, 05)$, 
no teste de Qui-quadrado e de Fisher, analisadas anteriormente.

As capacidades funcionais $(p=0,210)$, o DP $(p=0,281)$ e DS $(p=0,839)$ da qualidade de vida não apresentaram um efeito significante sobre a probabilidade de ter sintomatologia ansiosa. Apenas a saúde mental global $(p=0,044)$ apresentou esse efeito significante com o modelo ajustado $(p=0,586)$. Assim, recorreu-se ao ajustamento de um novo modelo significante apenas com a variável saúde mental global $(p=0,004)$.

O DS da qualidade de vida $(p=0,186)$ não apresentou efeito significante sobre a probabilidade de ter sintomatologia depressiva. Apenas o gênero $(p=0,021)$ e 0 DP da qualidade de vida $(p=0,022)$ apresentaram esse efeito significante com o modelo ajustado $(p=0,974)$. Desta forma, recorreu-se ao ajustamento de um novo modelo significante apenas com as variáveis: gênero $(p=0,025)$ e DP da qualidade de vida $(p=0,013)$.

A saúde mental global $(p=0,206)$, e o DF $(p=0,919)$, DP $(p=0,109)$ e DS $(p=0,541)$ da qualidade de vida não apresentaram efeito significante sobre a probabilidade de ter comorbidade de sintomatologia ansiosa e depressiva. Apenas o gênero $(p=0,036)$ apresentou esse efeito significante com o modelo ajustado $(p=0,298)$. Assim, recorreu-se ao ajustamento de um novo modelo significante apenas com as variáveis: gênero $(p=0,026)$, saúde mental global $(p=0,064)$, e DP da qualidade de vida $(p=0,067)$. 
Apesar de as variáveis: saúde mental global e DP da qualidade de vida não apresentarem significância estatística $(p>, 05)$, o modelo ajustado final composto por estas duas variáveis juntamente com a variável gênero foi o que apresentou maior percentagem de classificações corretas, e melhor sensibilidade e especificidade, comparativamente ao modelo $(p=0,878)$ que incluía apenas as variáveis: gênero $(p=0,021)$ e saúde mental global $(p=0,011)$.

\section{DISCUSSÃO}

No presente estudo foram encontradas taxas de incidência de sintomas ansiosos e de sintomas depressivos entre pessoas com LCA, em contexto de reabilitação, de $55,9 \%$ e 52,9\%, respectivamente. Para além disto, também foi analisada a incidência da comorbidade entre estas duas sintomatologias, correspondendo a uma taxa de $41,2 \%$.

As taxas de sintomatologia tanto ansiosa como depressiva, neste estudo, não são semelhantes às taxas encontradas no estudo de referência ${ }^{10}$. Este apresentou uma percentagem de 30,8\% para sintomas ansiosos e $36,7 \%$ para sintomas depressivos. A divergência de resultados pode estar associada à diferença de dimensão da amostra, que foi bastante superior no estudo de referência, e ao tempo decorrido após a lesão, que no estudo de referência foi limitado a 4 meses. Neste sentido, é possível perceber que pode haver um agravamento destes sintomas com o passar 
do tempo, já que a incidência de sintomatologia é superior em indivíduos cujo tempo decorrido após a lesão é maior.

Através da análise dos resultados da associação entre variáveis foi possível verificar que a sintomatologia ansiosa está estatisticamente associada às variáveis: capacidades funcionais, conforme observado na literatura ${ }^{3,21}$; saúde mental global; e qualidade de vida no DP e DS. Em contrapartida, e sendo estes resultados congruentes com a literatura, houve variáveis que não estabeleceram uma associação estatisticamente significativa com a presente sintomatologia. Entre elas, estão: o gênero ${ }^{11,12}$, a idade ${ }^{11,12}$, a escolaridade, o estado civil, a situação face ao emprego, o tempo pós-lesão, o mecanismo da lesão, a gravidade da lesão, a localização da lesão ${ }^{11}$, o funcionamento cognitivo global ${ }^{20}$, a autonomia nas $\operatorname{AVD}^{11}$, a qualidade de vida global $^{3,19,20}$, e a qualidade de vida no DF e DA.

Relativamente à sintomatologia depressiva, neste estudo verificou-se que a presente sintomatologia está associada às variáveis: gênero, apesar da controvérsia por parte da literatura ${ }^{14,15}$; e qualidade de vida no DP e DS. No entanto, e de acordo com o referido pela literatura, algumas variáveis não estabeleceram uma associação significante com a presente sintomatologia. Entre elas, estão: a idade ${ }^{13-}$ 15, a escolaridade, o estado civil ${ }^{15}$, a situação face ao emprego, o tempo pós-lesão, o mecanismo da lesão, a gravidade da lesão, a localização da lesão ${ }^{14}$, o funcionamento cognitivo global ${ }^{15}$, as capacidades funcionais, a saúde mental 
global, a autonomia nas AVD, a qualidade de vida global $^{3,14,20,23}$, e a qualidade de vida no DF e DA.

No que diz respeito à comorbidade entre sintomatologia ansiosa e depressiva, neste estudo verificou-se que a presente sintomatologia está associada às variáveis: gênero; saúde mental global; e qualidade de vida no DF, no DP e no DS. Contudo, e à semelhança do que foi verificado para as restantes sintomatologias, algumas variáveis não estabeleceram uma associação significante com a presente sintomatologia. Entre elas, estão: a idade, a escolaridade, o estado civil, a situação face ao emprego, o tempo pós-lesão, o mecanismo da lesão, a gravidade da lesão, a localização da lesão, o funcionamento cognitivo global, as capacidades funcionais, a autonomia nas AVD, a qualidade de vida global, e a qualidade de vida no DA.

Para além da associação entre variáveis, também foi estudada a relação funcional entre a sintomatologia tanto ansiosa, como depressiva e comorbidade de sintomas ansiosos e depressivos e os fatores sociodemográficos, clínicos e de qualidade de vida que foram associados às presentes sintomatologias. Através da regressão logística foi possível obter o modelo que melhor explica a presença destes sintomas.

No modelo final respeitante à sintomatologia ansiosa, das variáveis que estabeleceram uma associação significante com esta sintomatologia, apenas a saúde mental global foi capaz de explicar a presença de sintomas ansiosos. Deste modo, a probabilidade de um indivíduo com LCA, em 
contexto de reabilitação, desenvolver sintomas ansiosos aumenta com alterações mais severas da saúde mental global.

Já no modelo final relativo à sintomatologia depressiva, das variáveis que estabeleceram uma associação significante com esta sintomatologia, apenas o gênero e O DP da qualidade de vida foram capazes de explicar a presença de sintomas depressivos. Assim, a probabilidade de um indivíduo com LCA, em contexto de reabilitação, desenvolver sintomas depressivos é superior sendo do gênero feminino e aumenta com a qualidade de vida mais baixa no DP.

Por último, no modelo final referente à comorbidade entre sintomatologia ansiosa e depressiva, das variáveis que estabeleceram uma associação estatisticamente significativa com esta sintomatologia, apenas o gênero, a saúde mental global e o DP da qualidade de vida foram capazes de explicar a presença de comorbidade de sintomas. Logo, a probabilidade de um indivíduo com LCA, em contexto de reabilitação, desenvolver comorbidade de sintomas ansiosos e depressivos é superior sendo do gênero feminino, e aumenta com alterações mais severas da saúde mental global e com a qualidade de vida mais baixa no DP.

De acordo com a revisão da literatura seriam esperadas outras associações que não se verificaram neste estudo. Estes desvios relativamente a estudos similares, poderão ser justificados com as limitações do estudo. Deste modo, os resultados apresentados devem ser lidos com cautela, tendo em consideração o método de amostragem utilizado neste 
estudo (amostragem por conveniência) que resultou num reduzido número de participantes e na heterogeneidade da amostra, e, também, tendo em consideração o fato de terem sido utilizadas, essencialmente, medidas de avaliação de autopreenchimento que podem ter levado os participantes ao relato das próprias experiências de forma tendenciosa ${ }^{2}$.

\section{CONCLUSÕES}

O presente estudo demonstra que pessoas com LCA em contexto de reabilitação podem apresentar sintomas ansiosos, depressivos ou comorbidade de sintomas ansiosos e depressivos, tendo em consideração que aqueles do gênero feminino e com percepção negativa tanto da saúde mental global quanto da qualidade de vida ao nível psicológico apresentam maior suscetibilidade para a presença destes sintomas.

Conclui-se que o reconhecimento das dimensões sociodemográficas, clínicas e de qualidade de vida na detecção de alterações emocionais após LCA pode ser determinante na identificação de fatores que contribuem para o desenvolvimento de sintomas ansiosos e depressivos.

Dado que estes sintomas têm tendência a piorar com o decorrer do tempo e influenciam negativamente os resultados de reabilitação, seria oportuno intervir sobre estas componentes emocionais, sempre que possível, na fase inicial do processo de reabilitação. Desta forma, o compromisso do indivíduo com LCA no processo de 
reabilitação não seria prejudicado. Adicionalmente, os fatores identificados que explicaram a presença de sintomatologia ansiosa, depressiva e de comorbidade entre estas sintomatologias poderão fornecer aos profissionais da área da reabilitação os indicadores necessários para a sinalização dos indivíduos com maior probabilidade de necessidade de intervenção e acompanhamento psicológico. Neste sentido, seria importante focar a intervenção na saúde mental global e na qualidade de vida ao nível psicológico, visto que estes foram os fatores que melhor explicaram a presença de sintomatologia em pessoas com LCA.

\section{AGRADECIMENTOS}

A todos os clientes do Centro de Reabilitação que se disponibilizaram para participar neste estudo.

\section{REFERÊNCIAS}

1.McDonald S. Emotions are rising: the growing field of affect neuropsychology. J Int Neuropsychol Soc 2017;23:719-31. https://doi.org/10.1017/S1355617717000844

2.Lezak M, Howieson D, Bigler E, Tranel D. Neuropsychological Assessment. 5. ed. New York: Oxford University Press; 2012.

3. Masskulpan P, Riewthong K, Dajpratham P, Kuptniratsaikul V. Anxiety and depressive symptoms after stroke in 9 rehabilitation centers. J Med Assoc Thai 2008;91:1595-1602. https://pubmed.ncbi.nlm.nih.gov/18972905/

4.Zaninotto A, Vicentini J, Fregni F, Rodrigues P, Botelho C, Lucia M, et al. Updates and current perspectives of psychiatric assessments after traumatic brain injury: a systematic review. Front Psychiatry 2016;7:1-14. https://doi.org/10.3389/fpsyt.2016.00095

5.Rodrigues A, Silva S, Batista I, Nunes B, Dias C. Médicos-Sentinela: o que se fez em 2012 (Tese). Lisboa: Instituto Nacional de Saúde Doutor Ricardo Jorge; 2014. 
http://repositorio.insa.pt/bitstream/10400.18/1954/1/Relatorio Medi cos-Sentinela 2012.pdf

6.Santos M, Agrela N. Traumatic brain injury in Portugal: progress in incidence and mortality. Brain Inj 2019;33:1552-5. https://doi.org/10.1080/02699052.2019.1658227

7.Sohlberg M, Mateer C. Cognitive rehabilitation - an integrative neuropsychological approach. 2. ed. New York: Guildford Press; 2001. 8. Associação Psiquiátrica Americana (APA). DSM-5, Manual de Diagnóstico e Estatístico das Perturbações Mentais. 5. ed. Lisboa: Climepsi Editores; 2014.

9. Williams W. Neuro-rehabilitation and cognitive behaviour therapy for emotional disorders in acquired brain injury. In: Wilson $\mathrm{B}$. Neuropsychological Rehabilitation: Theory and Practice. Lisse: Swets \& Zeitlinger; 2003. p.115-36.

10.Brands I, Verlinden I, Ribbers G. A study of the influence of cognitive complaints, cognitive performance and symptoms of anxiety and depression on self-efficacy in patients with acquired brain injury. Clin Rehabil 2018;33:327-34.

https://doi.org/10.1177/0269215518795249

11.Burton C, Murray J, Holmes J, Astin F, Greenwood D, Knapp P. Frequency of anxiety after stroke: a systematic review and metaanalysis of observational studies. Int J Stroke 2012;8:545-59.

https://doi.org/10.1111/j.1747-4949.2012.00906.x

12.Scholten A, Haagsma J, Cnossen M, Olff M, Beeck E, Polinder S. Prevalence of and risk factors for anxiety and depressive disorders following traumatic brain injury: a systematic review. J Neurotrauma 2016;33:1969-94.bhttps://doi.org/10.1089/neu.2015.4252

13.Juengst $S$, Kumar $R$, Wagner A. A narrative literature review of depression following traumatic brain injury: prevalence, impact, and management challenges. Psychol Res Behav Manag 2017;10:175-86. https://doi.org/10.2147/PRBM.S113264

14.Kouwenhoven S, Kirkevold M, Engedal K, Kim H. Depression in acute stroke: prevalence, dominant symptoms and associated factors. A systematic literature review. Disabil Rehabil 2010;33:539-56.

https://doi.org/10.3109/09638288.2010.505997

15. Ouimet M, Primeau F, Cole M. Psychosocial risk factors in poststroke depression: a systematic review. Can J Psychiatry 2001;46:819-28. https://doi.org/10.1177/070674370104600905

16.Barker-Collo S. Depression and anxiety 3 months post stroke: prevalence and correlates. Arch Clin Neuropsychol 2007;22:519-31. https://doi.org/10.1016/j.acn.2007.03.002

17.Knutson K, Rakowsky S, Solomon J, Krueger F, Raymont V, Tierney $\mathrm{M}$, et al. Injured brain regions associated with anxiety in vietnam veterans. Neuropsychologia 2013;51:686-94.

https://doi.org/10.1016/j.neuropsychologia.2013.01.003

18. Lyketsos C, Kozauer N, Rabins P. Psychiatric manifestations of neurologic disease: where are we headed? Dialogues Clin Neurosci 2007;9:111-24. https://doi.org/10.31887/DCNS.2007.9.2/clyketsos 
19.Tang W, Chen Y, Lu J, Liang H, Chu W, Mok V, et al. Frontal infarcts and anxiety in stroke. Stroke 2012;43:1426-8. https://doi.org/10.1161/STROKEAHA.111.640482

20.Ayerbe L, Ayis S, Wolfe C, Rudd A. Natural history, predictors and outcomes of depression after stroke: systematic review and metaanalysis. $\mathrm{Br} \mathrm{J}$ Psychiatry 2013;202:14-21. https://doi.org/10.1192/bjp.bp.111.107664

21.Fann J, Katon W, Uomoto J, Esselman P. Psychiatric disorders and functional disability in outpatients with traumatic brain injuries. Am J Psychiatry https://doi.org/10.1176/ajp.152.10.1493

$1995 ; 152: 1493-9$.

22.Rao V, Munro C, Rosenberg P, Ward J, Bertrand M, Degoankar M, et al. Neuroanatomical correlates of depression in post traumatic brain injury: preliminary results of a pilot study. J Neuropsychiatry Clin Neurosci https://doi.org/10.1176/appi.neuropsych.22.2.231

$2010 ; 22: 231-5$.

23. Hibbard M, Ashman T, Spielman L, Chun D, Charatz H, Melvin S. Relationship between depression and psychosocial functioning after traumatic brain injury. Arch Phys Med Rehabil 2004;85:S43-53.

https://doi.org/10.1016/j.apmr.2003.08.116

24.Cicerone K, Dahlberg C, Kalmar K, Langenbahn D, Malec J, Bergquist $\mathrm{T}$, et al. Evidence-based cognitive rehabilitation: recommendations for clinical practice. Arch Phys Med Rehabil 2000;81:1596-1615. https://doi.org/10.1053/apmr.2000.19240

25.Simões M, Freitas S, Santana I, Firmino H, Martins C, Nasreddine $\mathrm{Z}$, et al. Montreal Cognitive Assessment (MoCA): Manual de administração e cotação. Coimbra: Laboratório de Avaliação Psicológica, Faculdade de Psicologia e de Ciências da Educação da Universidade de Coimbra; 2008.

26.Serra A, Canavarro M, Simões M, Pereira M, Gameiro S, Quartilho $\mathrm{M}$, et al. Estudos psicométricos do instrumento de avaliação de qualidade de vida da organização mundial de saúde (WHOQOL-BREF) para português de Portugal. Psiquiatr Clín 2006;27:41-9.

http://hdl.handle.net/10316/21539

27. Mundt J, Marks I, Shear M, Greist J. The work and social adjustment scale: a simple measure of impairment in functioning. $\mathrm{Br}$ J Psychiatry 2002;180:461-4. https://doi.org/10.1192/bjp.180.5.461

28.Sales C, Moleiro C, Evans C, Alves P. Versão Portuguesa do COREOM: tradução, adaptação e estudo preliminar das suas propriedades psicométricas. Rev Psiq Clín 2012;39:54-9.

https://doi.org/10.1590/S0101-60832012000200003

29.Pais-Ribeiro J, Silva I, Ferreira T, Martins A, Meneses R, Baltar M. Validation study of a portuguese version of the hospital anxiety and depression scale. Psychol Health Med 2007;12:225-37.

https://doi.org/10.1080/13548500500524088 